\title{
The Application of Design Structure Matrix Optimization Method Based on Genetic Algorithm
}

\author{
Lefeng Sun, Jing An and Hongmiao Yu \\ Institute of Manned Space System Engineering, China Academy of Space Technology, Beijing
}

\begin{abstract}
Based on the analysis the disadvantage of present research in optimization of Design Structure Matrix (DSM), the paper proposes a new algorithm of optimizing the design structure matrix, which sets up the objective optimization function, designs the way of coding, crossover, mutation based on genetic algorithm. In addition, the paper constructs DSM of the design process of a spacecraft, and uses the new algorithm for optimizing the DSM. The optimization result reveals that the new optimization algorithm has advantage in objective function, efficiency in finding best result.
\end{abstract}

Keywords-genetic algorithm; information feedback; design structure matrix; product design

\section{INTRODUCTION}

Design Structure Matrix (DSM) is firstly proposed by Steward in 1981 as Matrix-based information flow analysis frame [1]. DSM is used to analyze and optimize the design process of a product. It can effectively reduce design information feedback, can speeding up design cycle, and finally enhances product quality .Due to the importance of DSM in information flow analysis and product design and development, many experts make a lot of works on DSM research.

Eppinger[2] and Smith[3] propose a kind of design model based on DSM, and make optimizing for the operation sequence of design mission. However, the dimension of DSM in their optimization works is less than 6. Carrascosa[4] use DSM to estimate the development time of a product and compare different product development sequence, but the research is still limited to low dimension DSM. optimization. Robert [5] introduces work transfer matrix based on DSM that can find out the design activity which has the greatest impact on design process. Meanwhile, the model is still failed to use in optimizing high dimension DSM. Altus [6] proposes a method to deal with the sequence optimization and subsystem decomposing problem. $\mathrm{Xu}[7]$ proposes the steps of constructing DSM and the method of integrating models, and uses genetic algorithm to cluster DSM. However, the optimization method has disadvantage in object function choosing and optimization performance. Altogether, DSM is widely used in many areas such as spacecraft product design, telecommunication, and auto industry, etc. [8][9][10][11]

Based on the research in the past, the paper takes the genetic algorithm as optimization tool, and establish the optimization object function, design the selection, cross and mutation mechanism. Taking DSM of a spacecraft for example, optimization result shows that the optimization algorithm can solve the big scale DSM optimization problem, and has excellent performances

\section{INTRODUCTION TO A NEW DSM OPTIMIZATION ALGORITHM}

\section{A. Optimization Object}

A basic 0-1 type DSM is a square matrix .The cater-corner elements of DSM represent the design activities, usually is the subsystem of a product system. Out of the cater-corner use 0 or 1 to represent the relation between different elements.1 represents there are relation between two elements, 0 represents there are no relation between two elements. Taking the catercorner of DSM as a partition. The top right corner element of DSM represents the input flow, and the bottom left corner element of DSM represents the output flow (information feedback).Assuming the design process of a product includes four activities or subsystems: A, B, C, D. Based on the relations among elements, the DSM of the product can be show as Tab.1.The optimization object is the number of 1 in the bottom left corner of DSM should be as fewer as possible.

TABLE I. DSM OF A PRODUCT

\begin{tabular}{|c|c|c|c|c|}
\hline & A & B & C & D \\
\hline A & A & 1 & 0 & 1 \\
\hline B & 1 & B & 1 & 0 \\
\hline C & 0 & 1 & C & 1 \\
\hline D & 1 & 0 & 1 & D \\
\hline
\end{tabular}

B. Optimization Function

Comparing with other research [12], the paper takes the fitness function of DSM as Eq.1.

$$
T=\left(\left(\operatorname{wn} \times \sum_{i=1, t<j}^{n} \sum_{j=2}^{n} N_{i j}\right)+w c n \sum C N\right) \times\left(\sum_{i=1, t<j}^{n} \quad \sum_{j=2}^{n}(j-i) N_{i j}\right)(1)
$$

$\mathrm{N}$ represents information feedback point.CN represents cross point. D represents information feedback distance

$$
D=\left(\mathrm{wn} \times \sum_{i=1, t<j}^{n} \quad \sum_{j=2}^{n} N_{i j}\right)
$$

So Eq.1 can be showed as Eq.3 


$$
T=\left(\left(\mathrm{wn} \times \sum_{i=1, t<j}^{n} \quad \sum_{j=2}^{n} N_{i j}\right)+w c n \sum C N\right) \times D
$$

wn is the information feedback coefficient which can be choose in $[0,1]$. wcn is cross feedback coefficient which can be choose in [0,1].wn + ${ }^{\mathrm{wcn}}=1, N_{i j}$ is determined by Eq.4

$$
N_{i j}= \begin{cases}1 & D S M_{i j}>0, t<j \\ 0 & e l s e\end{cases}
$$

If wn smaller than wcn, the fitness function is more sensitive to cross point than information feedback point .That means optimization process should reducing the number of cross point seriously. If wcn smaller than wn, the fitness function is more sensitive to information feedback point than cross point. That means optimization process should reduce the number of information feedback point seriously. The information feedback point and cross point can be explained as Figure I.

$$
\text { A } \quad \text { B } \quad \text { C } \quad \text { D } \quad \text { E }
$$

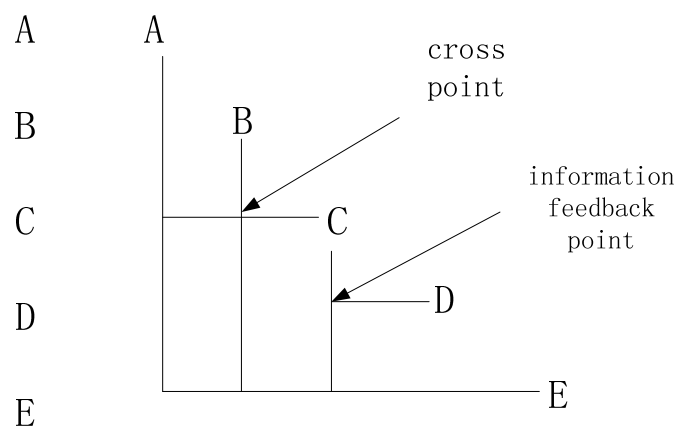

FIGURE I. DEFINITION OF INFORMATION FEEDBACK POINT AND CROSS POINT

\section{Coding Mode}

The optimization of DSM can be treated as ordering the sequence of design activities. Each sequence means a solution. The paper uses real number coding mode. Suppose the number of design activities is $n$. Then the design activities in DSM are marked from 1 to $\mathrm{n}$ before optimization. In the optimization process, the sequence of design activities can be change until reaching the best solution.

\section{Selection Mechanism}

According to the fitness function in Eq.1, the best solution means the minimum fitness. Meanwhile the best coding sequence is a few little different from the second best sequence. The new optimization algorithm adopts the excellence maintance strategy. In each generation, choosing the best 50 individuals to replace with the worst 50 individuals before cross and mutation operation.

\section{E. Cross Mechanism}

In the new algorithm, the generation satisfies the genetic coding condition, and each design activity is only appear once time in the coding. Choosing the truncation point stochastically, and taking the front part of one father gen as the front part of child gen in next generation. Meanwhile removing the child gen front number in the other father gen, and taking the new order number of the other father gen as the back part of child gen.

\section{F. Mutation Mechanism}

The mutation in DSM optimization algorithm means coding number changing stochastically. In the new algorithm, two number of gen are selected at random. And the two number exchange position in the gen. The mutation can be seen in Figure II.

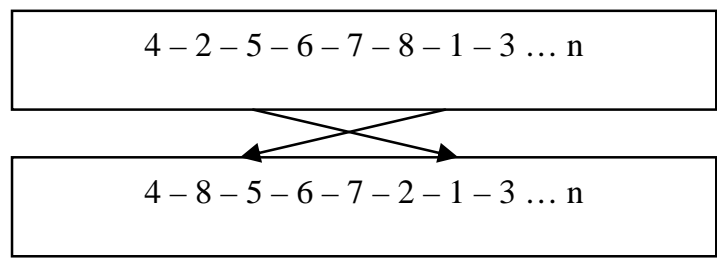

FIGURE II. MUTATION OF NEW ALGORITHM

\section{ApPLICATION Of DSM OptIMIZATION ALGORITHM}

The design of a spacecraft includes 13 design activities. The

\begin{tabular}{|c|c|c|c|c|c|c|c|c|c|c|c|c|c|}
\hline$\star$ & A1. & $\mathrm{A} 2$. & A3. & A4. & A5. & A6. & A 7. & A8. & A9. & $\mathrm{A} 10$ & A11. & $\mathrm{A} 12$ & $\mathrm{~A} 13$ \\
\hline A1. & A1. & 0. & 1. & 1. & 0. & 1. & 0. & 0. & 0. & 0. & 0. & 1. & 0. \\
\hline A.2. & 1 & $\mathrm{~A} 2$. & i. & 1. & 0. & 1. & 0. & 1. & 0. & 0. & 0. & 0. & 0. \\
\hline A3. & $\begin{array}{l}0 . \\
0\end{array}$ & 1. & A3. & 0. & 0. & 0. & 0. & 1. & 0. & 0. & 0. & $\vdots 0$. & 1. \\
\hline A4. & 1. & 0. & 0. & A4. & 0. & 0. & 0. & 1. & 1. & 0. & 0. & 0. & 1. \\
\hline A5. & : & 1 & 10 & 0. & As. & 0. & 0. & 0. & 0. & 0. & 0. & 0. & 0. \\
\hline A6. & $\vdots 0$. & 0. & 0. & 0. & 0. & A6. & 0. & 0. & 0. & 0. & 0. & $\vdots 0$. & 0. \\
\hline A 7. & 0. & 0. & 0. & 1. & 0. & 0. & A 7 . & 0. & 0. & 0. & 0. & $\vdots 0$. & 0. \\
\hline A8. & 1. & 0. & 0. & 0. & 0. & 0. & 0. & A8. & 0. & 0. & 0. & $\vdots 0$. & 0. \\
\hline A9. & 0. & 0. & 0. & 0. & 0. & 0. & 1. & 0. & A9. & 0. & 0. & $\vdots^{0 .}$ & 0. \\
\hline $\mathrm{A} 10$. & {$\left[\begin{array}{c}0 . \\
\hdashline .0\end{array}\right.$} & 0. & 0. & 0. & 0. & 0. & 0. & 0. & 1 & A10. & 1. & $\vdots 0$. & 0. \\
\hline A11. & 0. & 0. & 0. & 0. & 0. & 0. & 0. & 0. & 0. & 0. & A11. & 0. & 0. \\
\hline $\mathrm{A} 12$ & 0. & 0. & 0. & 0. & 0. & 0. & 0. & 0. & 0. & 0. & 0. & A 12 . & 0. \\
\hline A13. & 0. & 0. & 0. & 0. & 0. & 0. & 0. & 0. & 0. & 0. & 0. & 0. & $\mathrm{~A} 13$ \\
\hline
\end{tabular}
DSM of the spacecraft is showed as Table II.

TABLE II. DSM OF A SPACECRAFT

In the Table II, A1 represents shape design; A2 represents control and dynamic subsystem design; A3 represents energy subsystem design; A4 represents information subsystem design; 
A5 represents mission program; A6 represents rendezvous and docking; A7 represents communication subsystem design; A8 represents on-orbit construction; A9 represents extravehicular activity; A10 represents structure subsystem design; A11 represents space environment; A12 represents environment defense; A13 represents payloads. Can be seen from Table II. The number of 1 in the bottom left corner of DSM is 9.The population number is 500.The cross gene is 0.9.The mutation gene is 0.05 . The general generations are 1000 , $w n=0.5$, wn=wcn. The optimization result is show in Table III.

\section{TABLE III. OPTIMIZATION RESULT OF THE SPACECRAFT DSM}

\begin{tabular}{|c|c|c|c|c|c|c|c|c|c|c|c|c|c|}
\hline " & A5. & A7. & A10. & A2. & A3. & A4. & A11. & A1. & AG. & A12. & A13. & A6. & A8. \\
\hline AS. & AS. & 0. & 0. & 1. & 1. & 0. & 0. & 0. & 0. & 0. & 0. & 0. & 0. \\
\hline A7. & 0. & A7. & 0. & 0. & o. & 1. & 0. & 0. & 0. & 0. & 0. & 0. & U. \\
\hline A10. & 0. & 0. & A10. & 0. & 0. & 0. & 1. & 0. & 1. & 0. & 0. & 0. & 0. \\
\hline$A 2$. & 0. & 0. & 0. & $A 2$. & 1. & 1. & 0. & 1. & 0. & 0. & 0. & 1. & 1. \\
\hline A3. & 0. & 0. & 0. & a. & A3. & 0. & 0. & 0. & $\dot{p}$. & 0. & 1. & 0. & 1. \\
\hline A4. & 0. & 0. & 0. & $b$ & 0. & A4. & 0. & 1. & 1. & 0. & 1. & 0. & 1. \\
\hline A11. & 0. & 0. & 0. & $p$ & 0. & 0. & A11. & 0. & p. & 0. & 0. & 0. & 0. \\
\hline A1. & 0. & 0. & 0. & 0 & 1 & 1 & 0. & A1. & b. & 1. & 0. & 1. & 0. \\
\hline A9. & 0. & 0. & 0. & 0 & 0 & 0.1 & 0. & 0. & A9. & 0. & 0. & 0. & 1. \\
\hline A12. & 0. & 0. & 0. & 0. & 0. & 0. & 0. & 0. & 0. & A12. & 0. & 0. & 0. \\
\hline A13. & 0. & 0. & 0. & 0. & 0. & 0. & 0. & 0. & 0. & 0. & A13. & 0. & 0. \\
\hline A6. & 0. & 0. & 0. & 0. & 0. & 0. & 0. & 0. & 0. & 0. & 0. & A6. & 0. \\
\hline A8. & 0. & 0. & 0. & 0. & 0. & 0. & 0. & 1. & 0. & 0. & 0. & 0. & AB. \\
\hline
\end{tabular}

As Table III showed, The number of 1 in the bottom left corner of DSM is reduced to 4.Meanwhile the cross point and information feedback point in Table III is observably less than that in Table II. The optimization results shows that the new DSM optimization algorithm has excellent performance in optimizing DSM.

\section{SUMMARY}

The paper proposes a new algorithm of optimizing the design structure matrix, which sets up the objective function, designs the way of coding, crossover, mutation based on genetic algorithm. In addition, the paper constructs DSM of the design process of a spacecraft, and uses the new algorithm for optimizing the DSM. The optimization result reveals that the new optimization algorithm has advantage in objective function, efficiency in finding best result. The research makes the base for further application in other product's DSM optimization.

\section{REFERENCES}

[1] Steward, D. V. Systems Analysis and Management: Structure, Strategy and Design [ M ] . PBI: New York, 1981.

[2] Eppinger , Steven D. . Model based Approaches to Managing Concurrent Engineering [J]. Journal of Engineering Design , 1991, 2( 4) : 283- 290.
[3] Smith, Robert P. and Eppinger, Steven D. . A Predictive Model of Sequential Iteration in Engineering Design [J]. Management Science, 1997, 43(8): 1104- 1120.

[4] Carrascosa, M, Eppinger, S. D, and Whitney, D. E. .Using the Design Structure to Estimate Product Development Time [ C ] . Proceeding of DETC '98, 1998 ASME Design Engineering Technical Conference, Atlanta, Georgia, USA, 1998, Sept: 13- 16.

[5] Robert P. Smith, Steven D. Eppinger . Identifying Controlling Features o f Engineering Design Iteration [J] .Management Science, 1997, 43(3) : 276- 292.

[6] Altus, S. S, Kroo, I. M, and Gage, P. J. . A Genetic Algorithm for Scheduling and Decomposition of Multidisciplinary Design Problems [J]. Transactions of the ASME, 1996, 118(4): 486- 489.

[7] Xu Xiaogang. The Research on DSM and its Application in design management [D].Chongqing, Chongqing Univerity,2002.

[8] Melannie Hatch, Ralph D Badinelli. “A Concurrent Optimization Methodology for Concurrent Engineering”,IEEE Trans.on Engineering Management,Vol.46,No.1,1999,72-86.

[9] Eppinger,Steven D.,Whitney,Daniel E.,Smith,Robert and Gebala,David."A Model-based Method for Organizing Tasks in Product Development,"Research in Engineering Design,Vol.6, No.1,pp.1$13,1994$.

[10] Huovila,Pekka."Use of the Design Structure Matrix in Construction”, Proceedings of the Third International Workshop on Lean Construction,Albuquerque,NM,Oct.16-18,1995

[11] Pikosz,P.,Malmqvist,Johan.A Comparative Study of Engineering Change Management in Three Swedish Engineering Companies In Proceedings of DETC'98,Paper No DET98/EIM5684,Atlanta,GA,USA,1998.

[12] Sheng Haitao, Wei Fajie. The Research and Application of Geneticbased Design Structure Matrix Optimization Algorithm [J].Chinese Journal of Management Science, 2007, 15(4):98-104 